\section{Sensibilidad y especificidad de la prueba cutánea por punción con dos concentraciones del extracto estandarizado de Culex quinquefasciatus en niños alérgicos}

Raúl L Castro-Almarales ${ }^{1}$, Mirta Álvarez-Castelló2, Mercedes Ronquillo-Díaz², José S Rodríguez-Canosa ${ }^{2}$, Mayda González-León ${ }^{3}$, Bárbara I Navarro-Viltre ${ }^{4}$, Daniel Betancourt-Mesia ${ }^{5}$, Irene Enríquez-Domínguez ${ }^{2}$, Mary Carmen ReyesZamora $^{6}$, Yunia Oliva-Díaz ${ }^{1}$, Maytee Mateo-Morejón ${ }^{1}$, Alexis Labrada-Rosado ${ }^{1}$

\section{Resumen}

ANTECEDENTES: las opciones diagnósticas de las reacciones inmunológicas a la picadura del mosquito son limitadas. En Cuba, las reacciones mediadas por IgE más frecuentes son por picadura de Culex quinquefasciatus.

OBJETIVO: determinar la sensibilidad y especificidad de la prueba cutánea por punción con dos dosis del extracto estandarizado en unidades de nitrógeno proteico (UNP) de Culex quinquefasciatus (BIOCEN, Cuba).

MATERIAL Y MÉTODO: estudio analítico efectuado en 100 niños entre 2 y 15 años de edad: 50 pacientes atópicos con antecedentes de alergia a la picadura de mosquito e IgE sérica específica positiva a Culex quinquefasciatus y 50 pacientes atópicos sin antecedentes de alergia a la picadura de mosquito e IgE sérica específica negativa a Culex quinquefasciatus. La prueba cutánea por punción se realizó por duplicado en los antebrazos de los pacientes. Las dosis investigadas fueron 100 y $10 \mathrm{UNP} / \mathrm{mL}$.

RESULTADOS: en la prueba cutánea por punción con el extracto de mayor concentración se obtuvo un tamaño del área del habón de 22.09 $\mathrm{mm}^{2}$ y con la menor concentración de $8.19 \mathrm{~mm}^{2}$; una diferencia estadísticamente significativa ( $\mathrm{p}=0.001$, prueba t de Student). La prueba cutánea positiva se correlacionó en el $100 \%$ de los pacientes con la existencia de IgE específica. La prueba con ambas dosis mostró 94\% de especificidad y $88 \%$ de sensibilidad.

CONCLUSIÓN: la alta coincidencia en el resultado de la prueba cutánea nos muestra que puede sustituirse la concentración del extracto a $100 \mathrm{UNP} / \mathrm{mL}$ por la de menor concentración, sin perder confiabilidad en el diagnóstico de sensibilización al mosquito Culex quinquefasciatus, utilizando ese método in vivo.

PALABRAS CLAVE: sensibilidad, especificidad, prueba cutánea por punción, Culex quinquefasciatus.

\footnotetext{
${ }^{1}$ Departamento de Alergenos, Centro Nacional de Biopreparados, Dirección de Investigaciones. Facultad de Ciencias Médicas General Calixto García Íñiguez, La Habana, Cuba.

2 Servicio de Alergología, Hospital Universitario General Calixto García, Facultad de Ciencias Médicas General Calixto García Iñiguez, Plaza de la Revolución, La Habana, Cuba.

${ }^{3}$ Policlínico Docente Pedro Fonseca, Punta Brava, La Lisa, La Habana, Cuba.

${ }^{4}$ Hospital General de Cataluña, Barcelona, España.

${ }^{5}$ Servicio de Alergología, Hospital Héroes del Baire, Nueva Gerona, Isla de la Juventud, Cuba.

${ }^{6}$ Servicio de Alergología, Hospital Policlínico Docente Rosa Elena Simeón, Bejucal, Mayabeque y ISCMPC Victoria de Girón, La Habana, Cuba.
}

Recibido: 9 de julio 2015

Aceptado: 20 de noviembre 2015

Correspondencia

Dr. Raúl Lázaro Castro Almarales

rcastro@biocen.cu

Este artículo debe citarse como Castro-Almarales RL, Álvarez-Castelló M, RonquilloDíaz M, Rodríguez-Canosa JS y col. Sensibilidad y especificidad de la prueba cutánea por punción con dos concentraciones del extracto estandarizado de Culex quinquefasciatus en niños alérgicos. Rev Alerg Méx. 2016 ene-mar;63(1):11-19. 


\section{Sensitivity and specificity of prick skin test with two concentrations of standardized extract of Culex quinquefasciatus in allergic children.}

Raúl L Castro-Almarales ${ }^{1}$, Mirta Álvarez-Castelló², Mercedes Ronquillo-Díaz², José S Rodríguez-Canosa ${ }^{2}$, Mayda González-León ${ }^{3}$, Bárbara I Navarro-Viltre ${ }^{4}$, Daniel Betancourt-Mesia ${ }^{5}$, Irene Enríquez-Domínguez ${ }^{2}$, Mary Carmen ReyesZamora $^{6}$, Yunia Oliva-Díaz ${ }^{1}$, Maytee Mateo-Morejón ${ }^{1}$, Alexis Labrada-Rosado ${ }^{1}$

\section{Abstract}

BACKGROUND: Diagnostic options for immune reactions to mosquito bites are limited. In Cuba, IgE-mediated reactions are frequently related to Culex quinquefasciatus bite.

OBJECTIVE: To determine the sensitivity and specificity of skin prick test with two doses of standardized extract in nitrogen protein units (PNU) of Culex quinquefasciatus (BIOCEN, Cuba).

MATERIAL AND METHOD: An analytical study was conducted on 100 children between 2 and 15 years old. Fifty atopic patients with a history of allergy to mosquito bite and positive specific serum IgE Culex quinquefasciatus and fifty atopic patients without a history of allergy to mosquito bite and negative specific serum IgE to Culex quinquefasciatus. Skin prick tests (SPT) were performed by duplicates on the forearms of the patients. Investigated doses were $100 \mathrm{PNU} / \mathrm{mL}$ and $10 \mathrm{PNU} / \mathrm{mL}$.

RESULTS: SPT with the highest concentration obtained a mean wheal size of $22.09 \mathrm{~mm}^{2}$ and for lower doses of $8.09 \mathrm{~mm}^{2}$, a statistically significant difference ( $p=0.001$, Student's t test). Positive skin test correlated in $100 \%$ of patients with the presence of specific IgE. Testing with both doses showed a $94 \%$ of specificity and $88 \%$ of sensitivity.

CONCLUSION: The diagnostic accuracy of SPT using both doses of standardized extract was similar, which justifies its use for diagnosis of sensitization to Culex quinquefasciatus in patients with symptoms of allergy to mosquito bite.

KEYWORDS: sensitivity; specificity; skin prick test; Culex quinquefasciatus

\footnotetext{
${ }^{1}$ Departamento de Alergenos, Centro Nacional de Biopreparados, Dirección de Investigaciones. Facultad de Ciencias Médicas General Calixto García Î̃iguez, La Habana, Cuba.

2 Servicio de Alergología, Hospital Universitario General Calixto García, Facultad de Ciencias Médicas General Calixto García Iñiguez, Plaza de la Revolución, La Habana, Cuba.

${ }^{3}$ Policlínico Docente Pedro Fonseca, Punta Brava, La Lisa, La Habana, Cuba.

${ }^{4}$ Policlínico Docente Capdevila, Boyeros, La Habana, Cuba.

${ }^{5}$ Servicio de Alergología, Hospital Héroes del Baire, Nueva Gerona, Isla de la Juventud, Cuba.

${ }^{6}$ Servicio de Alergología, Hospital Policlínico Docente Rosa Elena Simeón, Bejucal, Mayabeque y ISCMPC Victoria de Girón, La Habana, Cuba.
}

\section{Correspondence}

Dr. Raúl Lázaro Castro Almarales rcastro@biocen.cu

\section{ANTECEDENTES}

Existen más de 3,500 especies de mosquitos, de los que Aedes vexans, Aedes aegypti y Culex quinquefasciatus son las especies más comunes en el mundo. ${ }^{1-4}$ En Cuba, estas dos últimas especies son las más comunes. ${ }^{5}$ 
Las reacciones a la picadura de mosquito pueden ser inmunológicas o no inmunológicas. ${ }^{1}$ De las reacciones inmunológicas a la picadura del mosquito Culex quinquefasciatus, las mediadas por IgE son las más frecuentes. ${ }^{1}$ Estas reacciones pueden ser locales, pequeñas o grandes, y sistémicas, con o sin peligro para la vida. Algunos autores consideran que el término alergia a la picadura de mosquito debe reservarse únicamente cuando el paciente tenga reacciones locales grandes, atípicas o sistémicas. ${ }^{6-8}$

El diagnóstico de alergia a la picadura de mosquito se obtiene a través de una anamnesis minuciosa y un examen físico detallado, aunado a la detección in vivo o in vitro de IgE específica a los alergenos del mosquito. ${ }^{1,6,7}$

La prueba cutánea por punción es el método menos costoso, más rápido y práctico para detectar hipersensibilidad tipo I a los alergenos del mosquito. En la actualidad, en todo el mundo se dispone de diferentes extractos de mosquitos para uso diagnóstico por prueba cutánea. ${ }^{1}$

En el Centro Nacional de Biopreparados (BIOCEN), en Cuba, se produce un extracto del mosquito Culex quinquefasciatus, estandarizado a 200,000 unidades de nitrógeno proteico (UNP/ $\mathrm{mL}$ ), pero se desconoce la dosis óptima a administrar para obtener el máximo de sensibilidad y especificidad en adultos y en niños.

El objetivo de este estudio es determinar la sensibilidad y especificidad de la prueba cutánea por punción utilizando dos concentraciones del extracto alergénico estandarizado de Culex quinquefasciatus, de producción nacional (BIOCEN, Cuba), en niños alérgicos a la picadura de este mosquito.

\section{MATERIAL Y MÉTODO}

Estudio analítico, controlado y prospectivo para validar la prueba cutánea por punción utilizando dos dosis del extracto del mosquito Culex quinquefasciatus. Se realizó en 100 sujetos (grupo A: 50 pacientes atópicos con antecedentes de alergia a la picadura de mosquito e IgE sérica específica positiva a Culex quinquefasciatus y grupo B: 50 pacientes atópicos sin antecedentes de alergia a la picadura de mosquito e IgE sérica específica negativa a Culex quinquefasciatus), entre 2 y 15 años de edad que acudieron consecutivamente a la consulta de Alergología del Hospital Universitario General Calixto García y tres consultorios del médico familiar del Guatao, perteneciente al Policlínico Docente Pedro Fonseca, La Lisa, La Habana, en el periodo de marzo de 2011 a marzo de 2012. Se ocultaron los resultados cuantitativos de las determinaciones séricas de IgE específica al mosquito Culex quinquefasciatus, registrándose el dato después de realizada la prueba cutánea por punción. Los investigadores del laboratorio de BIOCEN hicieron una lista de todos los pacientes a realizarse la prueba cutánea por punción. Este proceso se realizó en el Departamento de Alergenos de BIOCEN por personal ajeno al estudio. Este listado fue abierto al finalizar el ensayo para asignar a los pacientes a su respectivo grupo.

\section{Prueba cutánea por punción}

A todos los pacientes incluidos se les realizó la prueba cutánea por punción por duplicado en ambos antebrazos con las dos concentraciones (100 y $10 \mathrm{UNP} / \mathrm{mL}$ ) del extracto alergénico del mosquito Culex quinquefasciatus, a 200,000 UNP/mL. En conjunto, se utilizaron dos controles: negativo (solución diluente de extractos alergénicos [VALERGEN]: solución tampón-fosfato que contiene fenol $0.4 \%$ y albúmina sérica humana $0.03 \%$ ) y positivo (solución de histamina $\mathrm{HCl}, 10 \mathrm{mg} / \mathrm{mL}$ ). La prueba cutánea por punción se realizó según los procedimientos descritos por Dreborg. ${ }^{9}$ La prueba se consideró positiva cuando se obtuvo un diámetro medio de habón igual o mayor a $3 \mathrm{~mm}$ con el extracto alergénico 
y negativa cuando sel diámetro medio del habón fue menor de $3 \mathrm{~mm}$. Para considerar válida la prueba, el diámetro medio del habón producido por la histamina debía ser igual o mayor a $3 \mathrm{~mm}$ y por el control negativo menor a $3 \mathrm{~mm}^{10}$

El tamaño medio (área) de la reacción fue la variable primaria. La misma se calculó mediante la siguiente expresión:

$$
A=\pi d^{2} / 4
$$

Donde d es el diámetro promedio del habón calculado según se describió.

Todos los pacientes permanecieron 30 minutos en la consulta después de realizada la prueba cutánea, de acuerdo con lo establecido en el protocolo.

\section{Procesamiento de la sangre y obtención del suero}

Para la obtención del suero se extrajeron 10 $\mathrm{mL}$ de sangre dos semanas antes de realizar la prueba cutánea por punción, se colectaron en tubos Corning de $10 \mathrm{~mL}$, identificados con el número de inclusión del paciente y se enviaron al Departamento de Alergenos de BIOCEN para su procesamiento y análisis. El suero se separó mediante centrifugación a 6,000 rpm durante 10 min y se conservó a $-20^{\circ} \mathrm{C}$ hasta su análisis.

\section{Determinación de la IgE específica}

Para la determinación del título de IgE alérgenoespecífico se utilizó un ensayo ELISA indirecto validado por el BIOCEN para la producción de extractos alergénicos, con antígeno fijado a la fase sólida. Se usaron placas MaxiSorp (Nunc) de 96 pozos recubiertas durante toda la noche a $4{ }^{\circ} \mathrm{C}$ con $100 \mu \mathrm{L}$ del extracto alergénico de Culex quinquefasciatus, disueltos en tampón de recu- brimiento (carbonato $0.034 \mathrm{~mol} / \mathrm{L}$-bicarbonato $0.015 \mathrm{~mol} / \mathrm{L}, \mathrm{pH}$ 9.6). Los resultados cuantitativos de la determinación sérica de IgE específica al mosquito no se registraron hasta después de realizada la prueba cutánea por los investigadores clínicos.

\section{Indicadores evaluados para caracterizar el ensayo}

La eficacia de la prueba se midió por los valores de sensibilidad, especificidad, eficiencia y valores predictivos del resultado positivo y negativo, respectivamente. Para el cálculo de estos valores se usaron las siguientes definiciones: ${ }^{11}$

Verdadero positivo (VP): pacientes atópicos con antecedentes de alergia a la picadura de mosquito por historia clínica e IgE sérica específica positiva a Culex quinquefasciatus, con prueba cutánea por punción positiva.

Falso negativo ( $F N)$ : pacientes atópicos con antecedentes de alergia a la picadura de mosquito por historia clínica e lgE sérica específica positiva a Culex quinquefasciatus, con prueba cutánea por punción negativa.

Verdadero negativo (VN): pacientes atópicos sin antecedentes de alergia a la picadura de mosquito por historia clínica e IgE sérica específica negativa Culex quinquefasciatus, con prueba cutánea por punción negativa.

Falso positivo (FP): pacientes atópicos sin antecedentes de alergia a la picadura de mosquito por historia clínica e IgE sérica específica negativa Culex quinquefasciatus, con prueba cutánea por punción positiva.

Sensibilidad (S): porcentaje de verdaderos positivos del total de personas enfermas.

$$
S=V P /(V P+F N) \times 100
$$


Especificidad (E): porcentaje de verdaderos negativos del total de personas no enfermas.

$$
E=V N /(F P+V N) \times 100
$$

Valor predictivo del resultado positivo $(\mathrm{VP}+)$ : porcentaje de personas enfermas del total de personas con resultados positivos.

$$
V P+=V P /(V P+F P) \times 100
$$

Valor predictivo del resultado negativo (VP-): porcentaje de personas no enfermas del total de personas con resultados negativos.

$$
V P-=V N /(V N+F N) \times 100
$$

Eficiencia (Ef): porcentaje del total de resultados verdaderos, ya sean positivos o negativos.

$$
E f=V P+V N /(V P+V N+F P+F N) \times 100
$$

Los cálculos del tamaño muestral se realizaron con la ayuda del paquete de programas para el diseño de experimentos Glaxo-Wellcome C4-SDP v1.1. El estadígrafo seleccionado para comparar la respuesta en ambos grupos, así como en relación con los controles fue la mediana y la media geométrica del logaritmo del área del habón, porque experiencias previas demostraron que la transformación logarítmica de los datos iniciales (área) se ajusta mucho mejor a una distribución normal. Al aceptar la normalidad de la distribución de la variable primaria en la población a estudiar, el error en determinar la sensibilidad o la especificidad con un error $\varepsilon$ dado puede estimarse con la siguiente expresión:

$$
n=(K \alpha s / \varepsilon) 2
$$

donde $\mathrm{K} \alpha$ es el factor de confianza para un nivel de confianza $\alpha$ y s es la desviación estándar $(\mathrm{s}=$ $[p(1-p)] 1 / 2)$. Se estimó que los valores de sensibilidad y especificidad se encontrarían entre 85 y
$90 \%$. Entonces, para $\alpha=0.05$ y $\varepsilon=10 \%$ (es decir, un intervalo de $95 \%$ de confianza igual a $\pm 10 \%$ del valor determinado), obtuvimos un valor de $\mathrm{n}$ entre 35 y 49 sujetos. Al tomar en cuenta estas consideraciones, se seleccionaron 50 pacientes para cada grupo de estudio.

\section{Análisis estadístico de los resultados}

Se evaluó la distribución estadística de cada variable para probar su ajuste a la normalidad mediante la prueba de Kolmogorov-Smirnov y de Shapiro-Wilks. Por tanto, para la variable reactividad cutánea que se ajustó a la normalidad se calculó la media geométrica y la mediana del área del habón, así como sus respectivos intervalos de confianza de $95 \%$ y se compararon las medias geométricas para los diferentes productos mediante una prueba t de Student de muestras pareadas, con nivel de significación $\alpha$ menor a 0.05. Se determinó el grado de correlación de Spearman del tamaño de la reacción entre diferentes productos, incluyendo los resultados de todos los pacientes alérgicos. Se calcularon los intervalos de confianza de 95\% (IC95\%) de los valores de sensibilidad, especificidad, valores predictivos y eficiencia con la expresión:

$$
I C 95 \%= \pm 1.96[V(1-V) / n] 1 / 2
$$

Donde $\mathrm{V}$ es el valor correspondiente de cada parámetro.

Para las variables demográficas y clínicas (edad, sexo, enfermedad registrada) que no se ajustaron a una distribución normal se utilizó la mediana y sus IC $95 \%$ en cada grupo; las comparaciones entre los grupos se realizaron con la prueba $U$ de Mann-Whitney.

El procesamiento de los datos se realizó mediante las funciones estadísticas del programa Microsoft Excel v7.0 y el paquete estadístico Statistica v4.0. 


\section{Consideraciones éticas}

El protocolo lo analizó y aprobó el Comité de Ética del Hospital Universitario General Calixto García. Se justificó la inclusión de pacientes atópicos sin antecedentes de alergia a la picadura de mosquito por historia clínica con IgE sérica específica negativa para poder determinar la especificidad. No se realizaron pruebas de provocación a la picadura como patrón de referencia, lo que constituye una limitación de nuestro estudio; aunque, como otros autores, se utilizó el antecedente de alergia a la picadura de mosquito por historia clínica y la determinación de IgE sérica. ${ }^{1,6-8}$ Se obtuvo el consentimiento por escrito de los padres o tutores de los pacientes, previa información de las particularidades y riesgos del estudio. Además, se obtuvo el consentimiento de los pacientes incluidos mayores de ocho años.

\section{RESULTADOS}

En el grupo de pacientes alérgicos con IgE sérica específica positiva a Culex quinquefasciatus (grupo A), 15 pacientes fueron del sexo masculino y 35 del femenino; en el grupo de alérgicos con IgE sérica específica negativa a Culex quinquefasciatus (grupo B), 14 eran varones y 36 mujeres. En el grupo A, la mediana de la edad fue 7.4 años, mientras en el grupo B fue 7.7 años. Las diferencias de estos parámetros entre los dos grupos no fueron estadísticamente significativas (p mayor de 0.05). Cuadro 1

En el grupo A, de 50 individuos, 44 mostraron respuestas cutáneas positivas con ambas concentraciones del extracto de Culex quinquefasciatus. Se obtuvieron respuestas cutáneas negativas en seis pacientes para las dos concentraciones investigadas.

En el grupo B, la mayoría de los pacientes mostró resultados cutáneos negativos para las dos
Cuadro 1. Características demográficas de los pacientes incluidos

\begin{tabular}{lccc}
\hline & $\begin{array}{c}\text { Grupo A } \\
\mathbf{n = 5 0}\end{array}$ & $\begin{array}{c}\text { Grupo B } \\
\mathbf{n = 5 0}\end{array}$ & $\begin{array}{c}\mathbf{p} \\
\text { (U de Mann- } \\
\text { Whitney) }\end{array}$ \\
& & & 0.84 \\
Sexo (M/F) & $35 / 15$ & $36 / 14$ & 0.92 \\
$\begin{array}{l}\text { Mediana de edad } \\
\text { (años) }\end{array}$ & 7.4 & 7.7 & \\
Intervalo (IC 95\%) & $2-15$ & $2-15$ & \\
\hline
\end{tabular}

concentraciones del extracto de Culex quinquefasciatus (Cuadro 2). Se observó 100\% de coincidencia en el diagnóstico entre las dos concentraciones investigadas ( $p$ menor de 0.001).

En la prueba cutánea por punción con el extracto de mosquito a $100 \mathrm{UNP} / \mathrm{mL}$ se registró una media geométrica del área del habón en los pacientes positivos del grupo A de $22.09 \mathrm{~mm}^{2}$ (IC 95\%: 23.05-29.54), mientras con la concentración a $10 \mathrm{UNP} / \mathrm{mL}$ fue de $8.19 \mathrm{~mm}^{2}$ (IC 95\%: 11.78-8.82). Figura 1

La diferencia observada fue estadísticamente significativa (p menor de 0.05). En relación con el habón producido por la histamina, las reacciones hacia las dos concentraciones fueron menores, la diferencia fue significativa ( $p$ menor de 0.001).

Para las dos concentraciones del extracto alergénico de Culex quinquefasciatus se obtuvo $88 \%$ de sensibilidad, $94 \%$ de especificidad y $91 \%$ de eficiencia en la prueba cutánea por punción. Además, el valor predictivo del resultado positivo fue de $94 \%$ y el del resultado negativo fue de

Cuadro 2. Resultados de la prueba por punción en ambos grupos de estudio con las diferentes dosis

\begin{tabular}{|c|c|c|c|c|}
\hline \multirow[t]{2}{*}{ Pacientes } & \multicolumn{2}{|c|}{ Mosquito 100} & \multicolumn{2}{|c|}{ Mosquito 10} \\
\hline & Positivos & Negativos & Positivos & Negativos \\
\hline Alérgicos & 44 & 6 & 44 & 6 \\
\hline No alérgicos & 3 & 47 & 3 & 47 \\
\hline Total & 47 & 53 & 47 & 53 \\
\hline
\end{tabular}




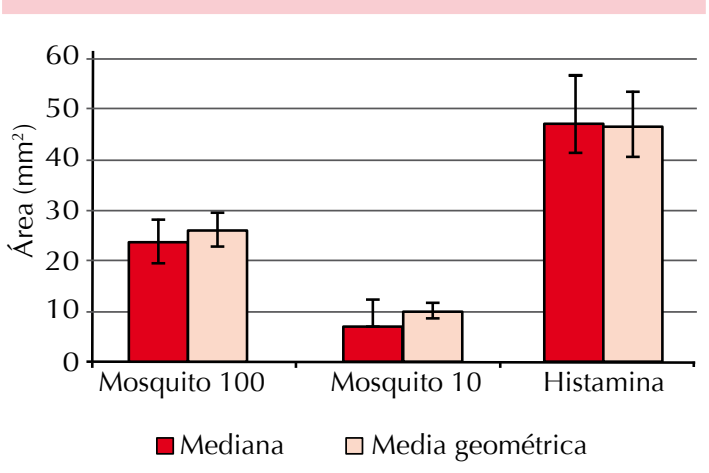

Figura 1. Mediana y media geométrica del área del habón (en $\mathrm{mm}^{2}$ ) en los pacientes positivos del grupo de alérgicos con IgE positiva para los diferentes productos utilizados.

89\% para ambas concentraciones investigadas (Figura 2). Estos valores resultaron superiores a los declarados inicialmente como aceptables en el protocolo.

En el transcurso del estudio no se produjeron eventos adversos.

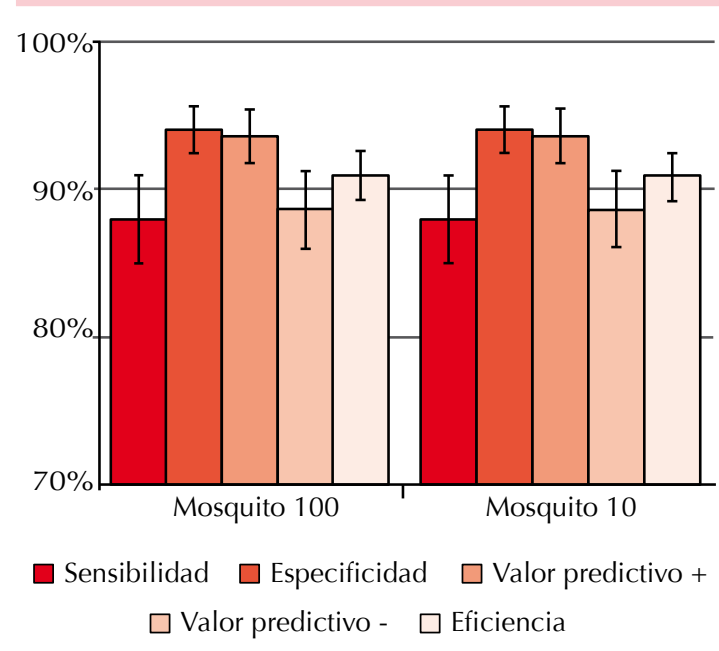

Figura 2. Resultados de las variables de eficacia.

\section{DISCUSIÓN}

En este estudio se obtuvo homogeneidad entre ambos grupos investigados, en relación con la edad y sexo. La preponderancia del sexo femenino concuerda con otros autores, que reportaron esta característica en estudios en los que se realizaron pruebas cutáneas por punción. ${ }^{12,13}$ La muestra poblacional es representativa de la población alérgica cubana, lo que permitiría la extensión de los resultados.

La diferencia encontrada en cuanto al tamaño de la reacción cutánea hacia las dos concentraciones coincide con lo reportado por otros autores. ${ }^{2,14,15}$ De manera inusitada hubo una coincidencia total entre los resultados cutáneos considerados positivos y negativos en ambos grupos para las dos concentraciones del extracto de Culex quinquefasciatus. El porcentaje de positividad en el grupo de pacientes alérgicos fue superior a 90\%; sin embargo, otros autores reportaron que las respuestas positivas se registran en 32\%. ${ }^{2,14,15}$ En esta investigación, la alta coincidencia en el resultado de la prueba cutánea nos muestra que la concentración del extracto a $100 \mathrm{UNP} / \mathrm{mL}$ puede sustituirse por la de menor concentración sin perder confiabilidad en el diagnóstico de sensibilización al mosquito Culex quinquefasciatus al usar ese método in vivo.

Si bien encontramos alta coincidencia en los resultados positivos entre las dos concentraciones investigadas, la de mayor concentración provocó reacciones de mayor tamaño en los pacientes alérgicos del grupo A que la de menor concentración. Esta diferencia podría considerarse una ventaja de la dosis mayor, porque indica mayor potencia alergénica y, por tanto, mayor sensibilidad potencial del diagnóstico. Otros autores expresan que a mayor potencia y concentración alergénica del extracto, su capacidad de detectar individuos sensibles es mayor. ${ }^{10,16-18}$ 
Los parámetros de eficacia para las dos concentraciones del extracto de Culex quinquefasciatus fueron mayores que los reportados (16 a 42\%) en otros estudios, ${ }^{6-8}$ lo que pudiera deberse al método diagnóstico seleccionado como referencia, que en este caso es el diagnóstico clínico basado en la aparición de síntomas relacionados con la picadura de mosquito y la determinación de IgE sérica específica a Culex quinquefasciatus. En Cuba, éste es el primer reporte de la sensibilidad y especificidad de dos concentraciones de un extracto del mosquito Culex quinquefasciatus. Los resultados permiten aseverar que la prueba cutánea por punción realizada con las dos concentraciones tiene sensibilidad y especificidad altas.

En el ensayo no se reportaron eventos adversos, lo que evidencia la seguridad de las concentraciones investigadas y de la prueba cutánea por punción, lo que coincide con reportes anteriores, que describen la ocurrencia de estos eventos como sumamente poco frecuentes en la prueba cutánea por punción. ${ }^{19,20}$ De modo que la evaluación de su frecuencia es posible solamente en estudios poscomercialización. ${ }^{10}$ Como reportaron otros autores, la prueba cutánea por punción es la prueba in vivo que ofrece menor riesgo para el paciente; es decir, la prueba cutánea por punción es muy segura y rara vez induce reacciones alérgicas sistémicas. .,10,19-21

\section{CONCLUSIÓN}

La prueba cutánea por punción con las concentraciones investigadas es eficaz, sensible y segura como herramienta diagnóstica de la alergia a la picadura del mosquito Culex quinquefasciatus. La alta coincidencia en el resultado de la prueba cutánea demuestra que la concentración del extracto a $100 \mathrm{UNP} / \mathrm{mL}$ puede sustituirse por la de menor concentración sin perder confiabilidad en el diagnóstico de sensibilización al mosquito Culex quinquefasciatus al utilizar ese método in vivo en los servicios de Alergología en Cuba.

\section{Agradecimientos}

El estudio en el que se basa este artículo lo financió en su totalidad el Centro de Biopreparados, entidad gubernamental de investigación-desarrollo de Cuba.

\section{REFERENCIAS}

1. Crisp HC, Johnson KJ. Mosquito allergy. Ann Allergy Asthma Immunol 2013;110:65-69.

2. Tulle MA. Mosquito-borne diseases. Curr Probl Pediatr Adolesc Health Care 2009;39:97-140.

3. Becker N, Petric D, Zgomba M, et al. Mosquitoes and their control. $2^{\text {nd }}$ ed. New York: Springer, 2010.

4. McKnight $\mathrm{S}$. What are the primary nuisance mosquitoes of North America? Wing Beats 2005;16:30-32.

5. Marquetti MC, Valdés V, Aguilera L, Navarro A. Vigilancia entomológica de Aedes (S) aegypti y otros culícidos en Ciudad de La Habana, Cuba 1991-1996. Rev Cubana Med Trop 2000;52: 133-137.

6. Peng $Z$, Simons FER. Mosquito allergy and mosquito salivary allergens. Protein Pept Lett 2007;14:975-981.

7. Asada H. Hypersensitivity to mosquito bites: a unique pathogenic mechanism linking Epstein-Barr virus infection, allergy and oncogenesis. J Dermatol Sci 2007;45:153-160.

8. Manrique MA, Gonzalez SN, Arias A, et al. Efficacy of immunotherapy with allergenic extract of Aedes aegypti in the treatment of large local reaction to mosquito bites in children. Ann Allergy Asthma Immunol 2011;107:A106.

9. Dreborg S, Frew A. Position paper: Allergen standardization and skin tests. Allergy 1993;48(Suppl. 14):49-82.

10. Demoly P, Pritte V, Bousquet J. In vivo methods for study of allergy: skin tests, techniques, and interpretation. In: Middleton E, Reed C, Ellis E, Adkinson N, Yunginger J, Busse W, editors. Allergy, Principles and Practice. $6^{\text {th }}$ ed. St Louis (Mo): Mosby Co., 2003;631-643.

11. Castro RL, Rodríguez J, Ronquillo M, Álvarez M y col. Sensibilidad y especificidad de la prueba cutánea por punción con extractos alergénicos estandarizados de Dermatophagoides pteronyssinus en adultos. Vaccimonitor 2013;22:24-29.

12. Ferrándiz R, Casas R, Dreborg S. Sensitization to Dermatophagoides siboney, Blomia tropicalis, and other domestic mites in asthmatic patients. Allergy 1996;51:501-505.

13. Castro ARL, Álvarez CM, Ronquillo DM, Rodríguez CJS, et al. Sensibilización a tres especies de ácaros en pacientes alérgicos de la zona costera de la ciudad de La Habana. Revista Alergia México 2009;56:31-35.

14. Peng Z, Simons FER. Comparison of proteins, IgE, and IgG binding antigens, and skin reactivity in commercial and laboratory-made mosquito extracts. Ann Allergy Asthma Immunol 1996;77:371-376. 
Castro-Almarales RL y col. Sensibilidad y especificidad de Culex quinquefasciatus

15. Peng $Z, X u$ W, James $A A$, et al. Expression, purification, characterization and clinical relevance of rAed a $1 \mathrm{da} 68-\mathrm{kDa}$ recombinant mosquito Aedes aegypti salivary allergen. Int Immunol 2001;13:1445-1452.

16. Bousquet J, Heinzerling L, Bachert C, Papadopoulos NG, et al. Practical guide to skin prick tests in allergy to aeroallergens. Allergy 2012;67:18-24.

17. Bernstein IL, Li JT, Bernstein DI, Hamilton R, et al. Allergy diagnostic testing: an updated practice parameter. Ann Allergy Asthma Immunol 2008;100:S1-S148.

18. Choi IS, Koh YI, Koh JS, Lee MG. Sensitivity of the skin prick test and specificity of the serum-specific IgE test for airway responsiveness to house dust mites in asthma. J Asthma 2005;42:197-202.

19. Norrman G, Falth-Magnusson K. Adverse reactions to skin prick testing in children prevalence and possible risk factors. Pediatr Allergy Immunol 2009;20:273-278.

20. Liccardi G, D’Amato G, Canonica GW, Salzillo A, et al. Systemic reactions from skin testing: literature review. J Investig Allergol Clin Immunol 2006;16:75-78.

21. Bernstein DI, Wanner M, Borish L, Liss GM. Twelveyear survey of fatal reactions to allergen injections and skin testing: 1990-2001. J Allergy Clin Immunol 2004;113:1129-1136. 